\title{
Desinfektion, Waschen, Kleben: Vermeiden von Infektionen auf der Intensivstation
}

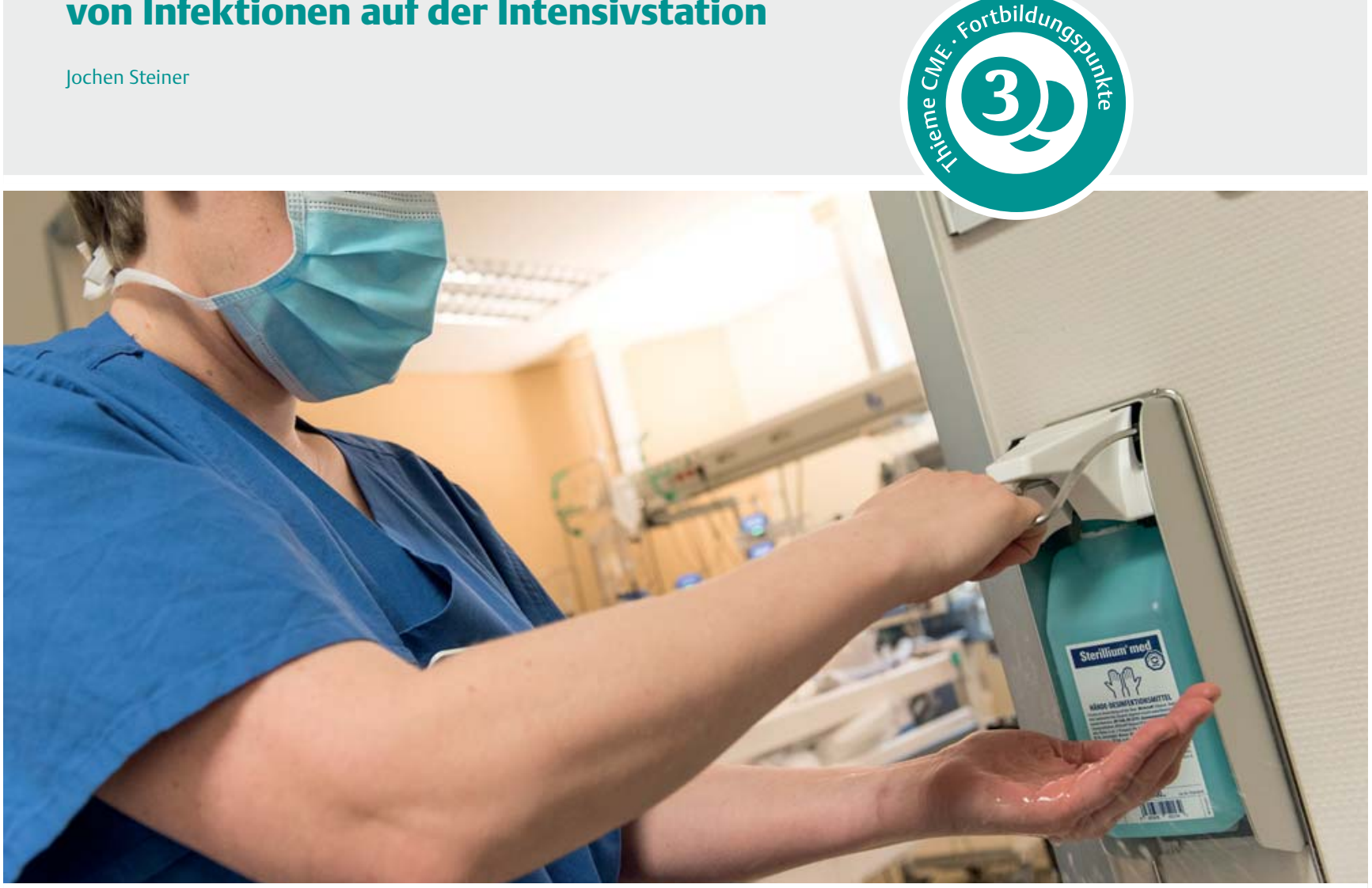

Quelle: KH Krauskopf.

Von den 400 000-600 000 Patienten mit nosokomialen Infektionen pro Jahr in Deutschland versterben ca. 10 000-15000 an deren Folgen. Vermutlich 20-30\% der nosokomialen Infektionen wären durch gezielte Maßnahmen vermeidbar [1]. Dies zeigt, wie dringend erforderlich hygienisch einwandfreies Arbeiten auf der Intensivstation ist. Dieser Beitrag zeigt die wichtigsten Maßnahmen zur Vermeidung nosokomialer Infektionen auf.

\section{Epidemiologie}

Die nosokomialen Infektionen stellen auf der Intensivstation weiterhin ein erhebliches Problem dar. Die Fallzahl in Deutschland wird pro Jahr auf 400000-600000 geschätzt. Die Gesamtprävalenz 2016 betrug 4,6\% (auf den Intensivstationen 17,07\%). Dabei waren am häufigsten die Infektionen der unteren Atemwege (24\%) aufgeführt, gefolgt von den postoperativen Wundinfektionen $(22,4 \%)$, den Harnwegsinfektionen $(21,6 \%)$ sowie der primären Sepsis (5,1\%). Von den Pneumonien waren $35,3 \%$ beatmungsassoziiert, von den Harnwegsinfektionen $60,7 \%$ mit einem Harnwegskatheter assoziiert. Von der primären Sepsis waren 78,1\% mit einem zentralen
Venenkatheter (ZVK) assoziiert (21,9\% mit einem peripheren Venenkatheter). Eine sekundäre Sepsis entwickelte sich in 26,6\% der Fälle aus einer Pneumonie, in 26,6\% aus einem Harnwegsinfekt sowie in $18,8 \%$ aus einer postoperativen Wundinfektion [2].

Merke

Die häufigsten nosokomialen Infektionen sind Infektionen der unteren Atemwege; ungefähr zwei Drittel der Harnwegsinfektionen sind Harnwegskatheterassoziiert. 


\section{Desinfizieren}

\section{Hände- und Flächendesinfektion}

Eine effektive, teils kausale und für den Patienten in keinster Weise belastende Maßnahme ist die Vermeidung der Keimübertragung. Ein bedeutender Faktor bei der Erregerübertragung sind die Hände. Die wichtigste Einzelmaßnahme der Basishygiene ist die hygienische Händedesinfektion. Sie sollte streng gemäß den Empfehlungen der Kommission für Krankenhaushygiene und Infektionsprävention (KRINKO) durchgeführt werden [3]. In Deutschland werden überwiegend alkoholbasierte Händedesinfektionsmittel verwandt. Je nach enthaltenem Alkohol (Ethanol, Propanol) ist die Mindesteinwirkzeit verschieden lang und muss nach Angaben des Herstellers exakt eingehalten werden. Die Hände müssen vor Anziehen der Handschuhe trocken sein, da Alkoholreste Materialschäden hervorrufen können und damit zur Patientengefährdung führen können. Bei Ethanol beträgt die Einwirkzeit für die chirurgische Händedesinfektion $120 \mathrm{~s}$, bei Propanol $90 \mathrm{~s}$. Ein Zusatz von remanent wirkenden Antiseptika (z.B. Chlorhexidin, Octenidin) besitzt keine Wirkungsverstärkung und erhöht zusätzlich die Gefahr einer Unverträglichkeit und einer Resistenzentwicklung [3].

\section{Merke \\ Vor und nach jedem Patientenkontakt, aber auch nach Kontakt mit der unmittelbaren Patienten- umgebung ist die hygienische Händedesinfektion durchzuführen.}

Denn bereits die Berührung von Oberflächen und Gegenständen aus der unmittelbaren, aber auch erweiterten Patientenumgebung führt zu einer relevanten Kontamination der Hände. Insofern ist es wichtig, dass die nähere Patientenumgebung, jedoch auch die Oberflächen adäquat gereinigt bzw. desinfiziert werden. In den Empfehlungen der KRINKO „Anforderungen an die Hygiene bei der Reinigung und Desinfektion von Flächen“ wird aufgeführt: Reinigungs- und Desinfektionsverfahren führen zu einer Verminderung von Mikroorganismen auf den behandelten Flächen. Die alleinige Reinigung erzielt eine ca. 50-80\%ige Reduktion, durch wirksame Desinfektionsverfahren kann die Effektivität auf mindestens 84-99,9\% erhöht werden. Desinfiziert werden müssen Flächen mit einem häufigen Hand- bzw. Hautkontakt, ebenso müssen die Fußböden in Bereichen mit besonderem Infektionsrisiko (Intensivstation) desinfiziert werden. Eine exakte Dosierung der Desinfektionsmittel ist Voraussetzung für eine wirksame Desinfektion. Es muss genügend Desinfektionsmittel auf die zu desinfizierende Fläche gelangen. Aus diesem Grund muss eine Scheuer-Wisch-Desinfektion („Nass-Wischen“) erfolgen.
Cave

Eine Sprühdesinfektion führt zu einer unzuverlässigen Wirkung und sollte nur im Ausnahmefall, wenn eine Scheuer-Wisch-Desinfektion von Flächen nicht möglich ist, angewandt werden [4].

Ein eher ernüchterndes Ergebnis zeigen die ComplianceBeobachtungen der „Aktion saubere Hände“ aus der Charité in Berlin: Die Compliance bei der Händedesinfektion in allen Funktionsbereichen lag im Jahr 2017 bei den Ärzten bei $49 \%$ und bei den Pflegekräften bei $65 \%$ (gepoolter arithmetischer Mittelwert) [5].

\section{Umgang mit Devices}

Neben der Übertragung von Krankheitserregern ist der Patient von Keimen besiedelt, die sich physiologischerweise am/im Körper befinden: auf der Haut, im Nasen-Rachen-Raum, im Gastrointestinaltrakt und Urogenitaltrakt. Zum Beispiel durch Anlage von Kathetern in das Gefäßsystem, den Urogenitaltrakt, den Respirationstrakt oder den Magen-Darm-Trakt kommt es zu Störungen der natürlichen Schutzbarrieren des Körpers (Haut, Schleimhaut). Dadurch können für den Körper sonst apathogene Keime diese Barrieren überwinden und sich das Device entlang - ausbreiten und lokale oder systemische Entzündungen hervorrufen. Eine Sonderstellung nimmt das zentrale Nervensystem (ZNS) ein. Das Gefäßendothel der Kapillaren des ZNS stellt durch seine besondere Anordnung der Endothelzellen mit seinen Tight Junctions sowie Perizyten und Astrozyten eine besondere Barriere dar. Dadurch ist es für Keime, allerdings auch für Medikamente, deutlich erschwert, sie zu passieren, um ins Gehirn zu gelangen. Eine Beschädigung dieser physiologischen Blut-Hirn-Schranke stellt z. B. die Anlage einer externen Liquordrainage (ventrikulär oder lumbal) dar.

Merke

Damit es nicht schon bei der Insertion von Kathetern zum Einschleppen von Keimen in den Körper kommt, ist die unbedingte Voraussetzung ein aseptisches Vorgehen.

Device-Anlage

Selbstverständlich ist vor der Punktion die entsprechende Stelle genügend großflächig mit einem Hautdesinfektionsmittel vorzubehandeln. Von der KRINKO wird ein schnell wirkendes alkoholisches Antiseptikum mit einem remanenten Antiseptikum empfohlen [6,7]. In Amerika kommt überwiegend Chlorhexidin zum Einsatz, ein weiteres remanentes Antiseptikum ist Octenidin. Beide Stoffe schädigen die Zellwand der Mikroorganismen und wirken so je nach Konzentration bakteriostatisch bzw. bakterizid. In einer Arbeit von Müller et al. war Octenidin dem Chlorhexidin in Bezug auf das antibakterielle Wirkungsspektrum überlegen und zeigte eine bessere Wirkung bei gleicher molarer Konzentration. Die in vitro gemessene antiseptische Aktivität ist bei Octenidin 3- bis 10-mal 
-Tab. 1 Erforderliches Material zur Device-Anlage.

\begin{tabular}{|l|l|l|l|l|}
\hline & ZVK & AK & EVD/LD & BK \\
\hline Mundschutz, Kopfhaube & + & $*$ & + & - \\
\hline sterile Handschuhe & + & + & + & + \\
\hline steriler Kittel & + & $*$ & + & - \\
\hline sterile Abdeckung & + & $*$ & + & + \\
\hline * erforderlich bei Anlage mittels Seldinger-Technik, AK: arterieller Katheter, \\
$\begin{array}{l}\text { BK: Harnwegskatheter, EVD: externe Ventrikeldrainage, LD: lumbale Liquor- } \\
\text { drainage, ZVK: zentraler Venenkatheter }\end{array}$
\end{tabular}

stärker als bei Chlorhexidin. Gleichzeitig ist der Biokompatibilitätsindex $(\mathrm{BI}=$ gleichzeitige antimikrobielle und zytotoxische Wirkung) je nach Keim mit 1,73 bzw. 2,11 deutlich höher als bei Chlorhexidin $(0,83$ bzw. 0,98$)$. Dies belegt die geringere zytotoxische Wirkung von Octenidin $[8,9]$.

Je nach Ort der Punktion und der Art des einzuführenden Katheters ist die Punktion und Katheteranlage unter maximalen Barrieremaßnahmen durchzuführen. Das bedeutet bei Anlage von zentralen Venenkathetern (s. „Praxis Zentraler Venenkatheter“), Liquordrainagen (s. „Praxis Periduralkatheter“) und externen Ventrikeldrainagen den Einsatz von Kopfhaube und Mundschutz, sterilem Kittel und sterilen Handschuhen. Ebenso erforderlich sind eine ausreichend große sterile Abdeckung im Bereich der Punktionsstelle sowie auch steriles Abdecken einer etwaigen Unterlage [6, 7]. Außer Kopfhaube, Mundschutz und sterilem Kittel sowie einem anderen Antiseptikum (Schleimhautdesinfektionsmittel) gilt bei der Anlage eines Harnwegskatheters das gleiche aseptische Vorgehen $[6,10]$ ( Tab. 1).

\section{PRAXIS}

\section{Zentraler Venenkatheter}

Die Vorbereitung einer ZVK-Anlage umfasst:

- Anlegen von Kopfhaube und Mundschutz

- ausgiebige Sprühdesinfektion der Punktionsstelle unter Beachtung der Mindesteinwirkzeit des Desinfektionsmittels (eine Applikation mittels Tupfer erbringt keinen Vorteil)

- Herrichten des sterilen Tisches (vorgefertigtes Set, ggf. Einzelkomponenten)

- hygienische Händedesinfektion

- Anziehen des sterilen Kittels und der sterilen Handschuhe (Hände müssen zuvor trocken sein)

- wenn der abzudeckende Bereich trocken ist: Abdecken der Punktionsstelle (z. B. mit Lochtuch)

- steriles Einpacken des Ultraschallkopfes und Kabels

\section{PRAXIS}

\section{Periduralkatheter (PDK)}

Die Vorbereitung einer PDK-Anlage umfasst:

- Anlegen von Kopfhaube und Mundschutz

- Lagern des Patienten

- Abtasten der Dornfortsätze und Festlegen der Punktionsstelle

- ausgiebige Sprühdesinfektion der Punktionsstelle unter Beachtung der Mindesteinwirkzeit des Desinfektionsmittels

- Herrichten des sterilen Tisches (vorgefertigtes Set, ggf. Einzelkomponenten)

- hygienische Händedesinfektion

- Anziehen des sterilen Kittels und der sterilen Handschuhe (Hände müssen zuvor trocken sein)

- wenn der abzudeckende Bereich trocken ist: Abdecken der Punktionsstelle (z. B. mit Lochtuch)

\section{Manipulationen an Devices}

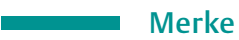

Auch für Manipulationen an den Kathetern gilt: zuvor und danach hygienische Händedesinfektion sowie Desinfektion der Devices vor jeder Manipulation.

Über die Art der Desinfektion bei Probenentnahme aus oder Injektion in die Katheter herrscht Uneinigkeit in der Literatur. Studien haben gezeigt, dass im Laufe der Liegedauer zwischen 5 und 20\% der Dreiwegehähne und Katheterhubs mikrobiell besiedelt sind. Die Vorschläge zur Desinfektion reichen vom Abreiben mit einem alkoholhaltigen Tuch bis hin zu Antiseptika sezernierenden Verschlussstopfen. Die KRINKO empfiehlt als eine Möglichkeit der Desinfektion die Sprühdesinfektion mit einem Hautantiseptikum und das Ausschütteln des Konus nach Ablauf der Einwirkzeit auf eine sterile Kompresse. Vor jeglichen Manipulationen an einem ZVK sowie mit ihm verbundenen Dreiwegehähnen muss eine hygienische Händedesinfektion sowie eine Desinfektion des Dreiwegehahns durchgeführt werden [7]. Eine strikte Einhaltung der hygienischen Händedesinfektion und die Desinfektion der dafür vorgesehenen Abnahmestelle an einer externen Ventrikeldrainage sind obligat.

Harnwegskatheter-Infektionen gehören zu den häufigsten nosokomialen Infektionen. Außer der strengen Indikationsstellung für die Anlage eines Blasenkatheters gehört zu den empfohlenen Präventionsmaßnahmen,

- die Katheterisierung aseptisch vorzunehmen,

- vor und nach jeder Manipulation am Blasenkatheter oder Drainagesystem eine hygienische Händedesinfektion durchzuführen und

- beim Wechsel des Ableitungssystems die Konnektionsstellen mit einem alkoholischen Präparat zu desinfizieren. 
Die Reinigung des Genitales sollte nur mit (Trink-)Wasser und Seifenlotion erfolgen, ohne Zusatz antiseptischer Substanzen. Eine Instillation von antiseptischen oder antimikrobiellen Substanzen in den Katheter zur Behandlung und Prävention katheterassoziierter Infektionen ist obsolet [10].

\section{Merke}

Für alle Devices gilt: Die Indikation muss täglich überprüft werden.

\section{FALLBEISPIEL}

\section{ZVK-assoziierte Infektion}

Bei einer 76-jährigen Patientin kommt es nach Hemihepatektomie bei synchron metastasierendem Rektumkarzinom zu einem protrahierten Intensivaufenthalt wegen SSI (postoperative Wundinfektion) und respiratorischer Insuffizienz bei vorbestehender COPD (chronisch obstruktive Lungenerkrankung). Nach 12 Tagen - 2 Tage vor geplanter Verlegung in die Reha - wird sie auf Normalstation verlegt. Bei schlechtem Venenstatus erfolgt die Verlegung mit ZVK (Liegedauer 12 Tage). 36 Stunden später ist die Rückübernahme der Patientin auf Intensivstation erforderlich wegen kardiorespiratorischer Instabilität (RR 76/34 mmHg, HF 148 bpm, $\mathrm{SpO}_{2}$ unter $6 \mathrm{I} \mathrm{O}_{2}$ Gabe $83 \%, \mathrm{AF} 45 / \mathrm{min})$, Fieber von $39,7^{\circ} \mathrm{C}$ und Vigilanzminderung. Die Patientin stabilisiert sich unter Volumen- und Vasopressorengabe. Nach Abnahme von Blutkulturen (über ZVK und Punktion) wird der ZVK entfernt und bei V. a. katheterassoziierte Infektion eine kalkulierte Antibiose begonnen. In den Blutkulturen (BK) wird Staphylococcus (S.) aureus nachgewiesen: Positiv-Meldung nach 6,5 h (BK über Punktion) und 6,8 h (BK über ZVK). An der ZVK-Spitze kann ebenfalls $S$. aureus nachgewiesen werden.

\section{Waschen}

\section{Ganzkörperwaschung}

Chlorhexidin ist ein Antiseptikum und wurde 1950 bei der Suche nach einem Malariamittel in England entwickelt. Es besitzt eine unspezifische antibakterielle Wirkung. In niedriger Konzentration wirkt es bakteriostatisch, in hoher bakterizid. Die Wirksamkeit beruht darauf, dass sich das positiv geladene Molekül an die negativ geladenen Komponenten der Bakterienzellwand bindet und zu einer Schädigung der äußeren Zellschichten führt. In niedriger Konzentration kommt es zu einer Veränderung des osmotischen Gleichgewichts der Bakterienzelle, dadurch treten Kalium und Phosphor aus der Zelle aus. So wird ein weiteres Wachstum verhindert (bakteriostatisch). Bei hoher Chlorhexidin-Konzentration kommt es zu Ausfäl- lung der zytoplasmatischen Bestandteile und damit zum Zelltod (bakterizid). Der maximale Effekt tritt bereits nach $20 \mathrm{~s}$ auf $[11,12]$.

Inzwischen ist Chlorhexidin weitverbreitet - von der Mundspüllösung über Händedesinfektionsmittel bis hin zur Ganzkörperwaschung und Infektionsprophylaxe bei Katheter-Eintrittsstellen sowie antimikrobiellen Beschichtung von intravasalen Kathetern.

Unter der Vorstellung, dass durch Chlorhexidin-Ganzkörperwaschungen die Keimzahl auf der Haut vermindert und dadurch die Häufigkeit einer katheterassoziierten Infektion verringert wird, wurden viele Studien durchgeführt. Die Ergebnisse sind sehr uneinheitlich (s. „Info Chlorhexidin-Körperwaschungen“).

INFO

\section{Chlorhexidin-Körperwaschungen}

Die Studie von Gastmeier et al. [13] zeigte: Auf medizinischen Intensivstationen und Knochenmarkstransplantationsstationen konnten die Anzahl der Blutstrominfektionen und die Anzahl der Infektionen mit Methicillin-resistentem S. aureus (MRSA) signifikant reduziert werden. Dies ließ sich jedoch auf operativen Intensivstationen nicht nachweisen. Als mögliche Ursache wurde eine höhere Anzahl sekundärer Blutstrominfektionen genannt. In einer Untersuchung von Frost et al. [14] wurde die Effektivität der Chlorhexidin-Ganzkörperwaschung zur Reduktion der katheterassoziierten Blutstrominfektionen und MRSA-Infektionen beschrieben. Noto et al. [15] konnten dies nicht bestätigen. Dies traf auch für in der Studie untersuchte Harnwegskatheter-assoziierte Infektionen, ventilatorassoziierte Pneumonien und Infektionen mit Clostridium difficile zu. In einem Review von 2019 von Lewis et al. [16] zeigte sich keine Evidenz dafür, dass Chlorhexidin-Körperwaschungen nosokomiale Infektionen, die Mortalität oder die Länge des Intensivaufenthalts reduzieren.

In unserer Klinik werden Ganzkörperwaschungen mit Chlorhexidin nach dem klinikeigenen Hygieneplan nur im Rahmen des Dekontaminierungsschemas bei MRSAKeimträgerschaft unter regelmäßigen Kontrollabstrichen eingesetzt.

\begin{abstract}
Merke
Für Chlorhexidin-Ganzkörperwaschungen wurde bislang keine Reduktion Device-assoziierter Blutstrominfektionen auf operativen Intensivstationen belegt. Sie sollten speziellen Indikationen (wie Dekontamination bei MRSA-Besiedelung) vorbehalten bleiben.
\end{abstract}

\section{Dekontamination von Oro- und Nasopharynx}

\section{Ventilatorassoziierte Pneumonie}

Unter den nosokomialen Infektionen nimmt die ventilatorassoziierte Pneumonie (VAP) einen hohen Stellenwert ein. Trotz Einhaltung aller Hygienemaßnahmen sind die 


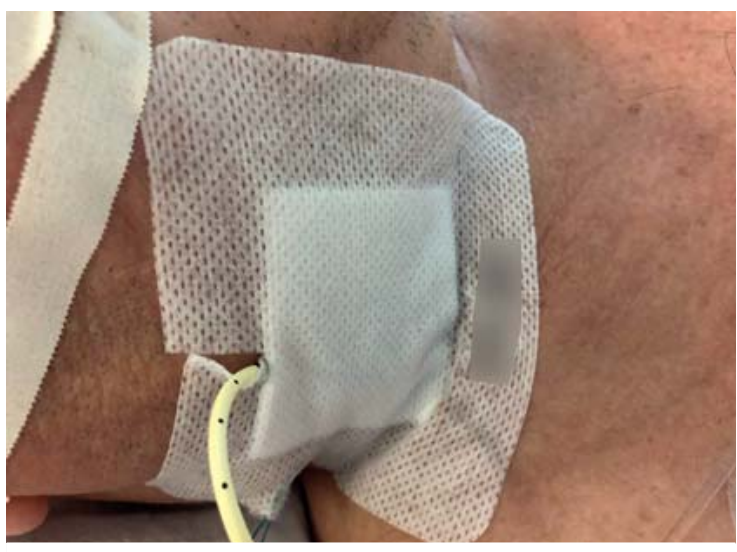

- Abb. 1 Gazepflasterverband.

Patienten durch ihre eigene Keimbesiedelung von Nasound Oropharynx, Magen und Darm bedroht. Insbesondere der Oropharynx ist eine häufige Quelle für pulmonale Infektionen. Ein weiteres Keimreservoir, vor allem für Methicillin-sensiblen S. aureus (MSSA) und MRSA, stellt die Nase dar. Um die Entstehung einer VAP zu verhindern, ist eine Reihe von Maßnahmen erforderlich.

\section{Merke}

Zum VAP-Präventions-Bundle gehört neben Endotrachealtuben mit subglottischer Absaugung und erhöhter Oberkörperlagerung (ca. $30^{\circ}$ ) auch die Dekontamination des Oropharynx.

Chlorhexidin ist das hierzu am häufigsten verwendete Antiseptikum. Bei Anwendung des gesamten Bundles konnte die Entstehung einer VAP signifikant reduziert werden. Eine prophylaktische Langzeitanwendung von Antibiotika im Sinne einer selektiven Darmdekontamination ist wegen der erhöhten Gefahr der Resistenzbildung umstritten [17].

\section{Offene herz- und lungenchirurgische Eingriffe}

Patienten, die sich einem offenen herzchirurgischen Eingriff unterziehen, erleiden in bis zu über $20 \%$ eine nosokomiale Infektion. Diese Operationen werden vermehrt an älteren, mit vielen Begleiterkrankungen vorbelasteten Patienten durchgeführt und es kommt hierbei häufiger zu einem verlängerten Intensivaufenthalt. Daher ist hier eine Infektionsprophylaxe besonders wichtig, vor allem die Eradikation von S. aureus, dem wichtigsten Erreger bei chirurgischen Wundinfektionen (SSI).

Segers et al. konnten zeigen: Durch Dekontamination von Naso- und Oropharynx mittels Chlorhexidin-haltiger Mundspüllösung und Chlorhexidin-haltiger Nasensalbe ließ sich eine effektive Reduktion der Infektionen der unteren Luftwege und der tiefen Wundinfektionen erzielen. Da Mupirocin einer der Grundpfeiler der MRSA-Behandlung ist, wurde auf dessen Einsatz verzichtet, weil man

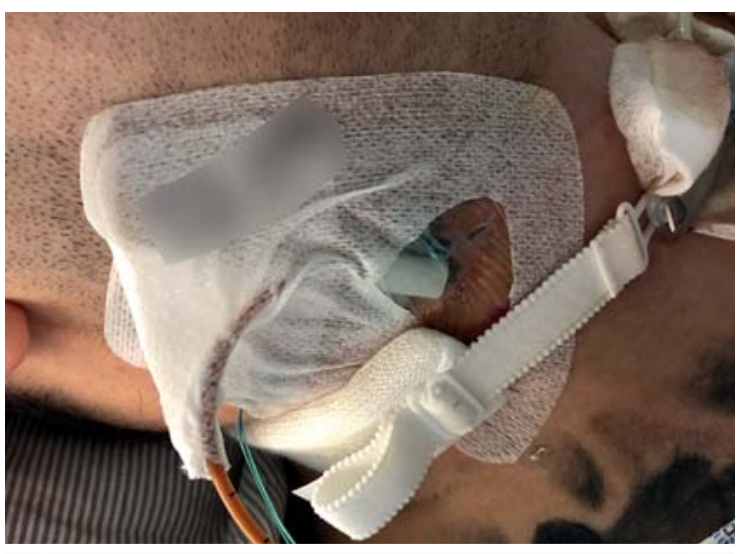

- Abb. 2 Semipermeabler Folienverband.

eine Resistenzbildung befürchtete. Patienten, die eine selektive Darmdekontamination erhielten, wurden wegen mehrfach beschriebener Resistenzentwicklungen von der Studie ausgeschlossen. Es zeigte sich, dass es unter Chlorhexidin-Gabe bei einigen Erregern zu einem Anstieg der minimalen Hemmkonzentration (MHK) kommen kann. Dies wurde von den Autoren jedoch nicht mit einer kompletten Resistenz der Keime gegen Chlorhexidin gleichgesetzt [18].

In einer Studie von D'Journo et al. wurden im Rahmen einer offenen Lungenchirurgie ebenfalls Naso- und Oropharynx mit Chlorhexidin dekontaminiert. Es zeigte sich eine signifikante Reduktion von Bakteriämie und SSI. Die Anzahl der Infektionen des Respirationstrakts, die Länge des Krankenhausaufenthalts, die Keimbesiedlung der Trachea und der postoperative Antibiotikaverbrauch wurden nicht beeinflusst [19].

Merke

Die Dekontamination von Naso- und Oropharynx soll nur bei Risikopatienten durchgeführt werden. Sie reduziert das SSI-Risiko.

\section{Kleben}

\section{Zentraler Venenkatheter}

Nicht antiseptische Verbände

Die Eintrittsstelle des ZVK muss vor Kontamination und daraus resultierend - drohender katheterassoziierter Infektion geschützt werden. Die dazu verwendeten Abdeckungen können nichtantiseptisch wirkende Verbände (sterile Gazepflaster oder semipermeable Folienverbände) sein ( $\triangleright$ Abb. 1 u. 2). Der Vorteil bei Gazepflastern besteht darin, dass austretendes Sekret/Blut sowie Schweiß aufgesaugt werden können. Ein Nachteil ist, dass keine direkte Kontrolle der Eintrittsstelle ohne Entfernung des Verbandes möglich ist. Die semipermeablen Folienver- 


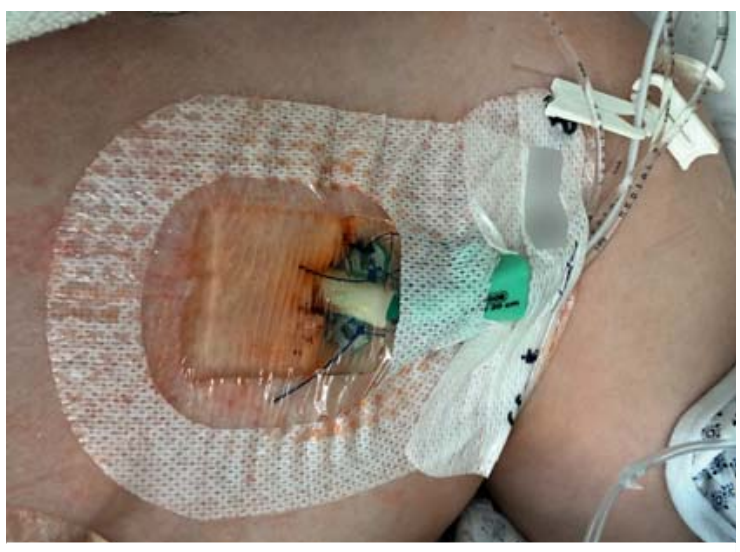

Abb. 3 Verband mit Chlorhexidin-Gelkissen.

bände ermöglichen zwar eine direkte visuelle Kontrolle der Eintrittsstelle, sind aber nicht in der Lage, Flüssigkeiten aufzusaugen. Damit können sich feuchte Kammern bilden, die ein ideales Milieu für das Keimwachstum darstellen [7].

\section{Antiseptische Verbände}

Eine andere Möglichkeit, die Katheter-Eintrittsstelle zu schützen, ist das Aufbringen von Verbänden, die aseptische Inhaltsstoffe abgeben ( $\bullet$ Abb.3). Auch hier zeigt sich eine uneinheitliche Studienlage. In einer Reihe von Studien konnte die präventive Wirkung von Verbänden mit Chlorhexidin-haltigem Gelkissen hinsichtlich einer Reduktion von katheterassoziierten Blutstrominfektionen nachgewiesen werden [20]. In anderen gab es keine signifikante Reduktion der katheterassoziierten Blutstrominfektionen [21]. In weiteren Studien konnte eine Reduktion auch mit Verbänden ohne aseptischen Wirkstoff mit nachhaltigem Effekt erzielt werden.

Die aktuellen britischen und US-amerikanischen Empfehlungen (mit Ausnahme der American Pediatric Surgical Association) beinhalten den Einsatz Chlorhexidin-haltiger ZVK-Verbände bei Hochrisikopatienten sowie bei anhaltend hohen Infektionsraten - aber erst nach Ausschöpfung aller anderen Maßnahmen. Diesen Empfehlungen schließt sich auch die KRINKO an [7].

\footnotetext{
Merke

Chlorhexidin-Pflaster sollen nur bei spezieller Indikation verwendet werden (z. B. Hochrisikopatienten). Sie ersetzen nicht die tägliche Kontrolle der DeviceEintrittsstelle!
}

Die Art des eingesetzten Verbandes ist aber auch abhängig von dem Ort der Katheter-Eintrittsstelle, der Art des Katheters, der Hautbeschaffenheit (trocken, schweißig, haarig). Eine starre Festlegung kann vorab nicht getroffen werden.

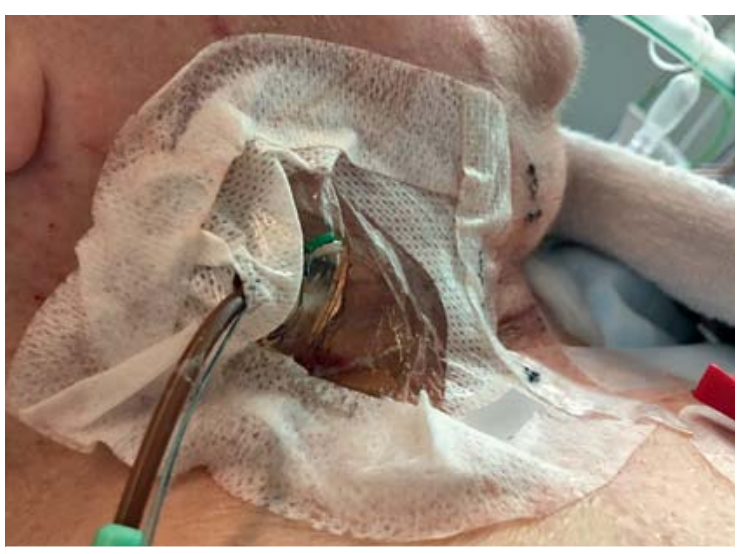

Abb. 4 Durchfeuchteter Folienverband.

\section{Verbandwechsel}

Ein Wechsel des Katheterverbands muss in regelmäßigen Intervallen erfolgen. Diese Intervalle differieren nach eigenen Erfahrungen von Klinik zu Klinik. In unserer Klinik gelten folgende Zeiträume für den Verbandwechsel:

- Gazepflaster jeden Tag

- Folienpflaster alle 4 Tage

- Pflaster mit Chlorhexidin-Gelkissen alle 7 Tage

Bei jedem Verbandwechsel sollte die Katheter-Eintrittsstelle mit einem alkoholbasierten Desinfektionsmittel mit einem Zusatz eines remanenten Antiseptikums desinfiziert werden.

\footnotetext{
Cave

Unabhängig von der Art des ZVK-Verbandes gilt: Jeder nicht mehr intakte, durchfeuchtete oder sonst verunreinigte Verband muss sofort erneuert werden ( Abb. 4) [7].
}

\section{Externe Ventrikeldrainage}

Es gibt zahlreiche Untersuchungen zu Liquorinfektionen bei einliegenden externen Ventrikeldrainagen (EVD). Diese äußern sich unterschiedlich zur Ursache in Abhängigkeit von der Liegedauer der EVD und den Liquor-Probenentnahmen sowie der Art der verwendeten Drainagekatheter. Auch werden Liquorinfektionen durch die Ursache des Liquoraufstaus sowie eine prophylaktisch verabreichte Antibiose beeinflusst [22].

Für die Prävention von Infektionen, die mit Gefäß- und Harnwegskathetern assoziiert sind, gibt es klare Empfehlungen. Aktuelle Leitlinien/Empfehlungen für die Infektionsprävention bei EVD liegen dagegen weder von der KRINKO, der Deutschen Gesellschaft für Neurochirurgie noch von der Deutschen Gesellschaft für Neurologie vor. 


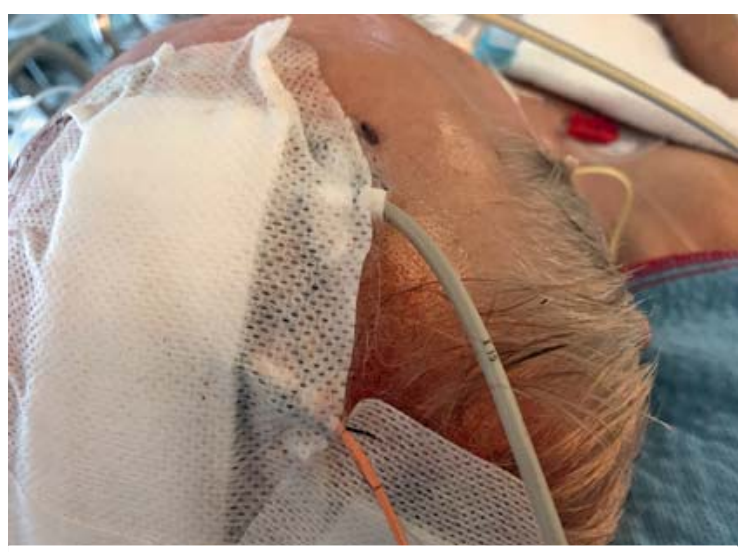

Abb. 5 Gazepflasterverband einer getunnelten externen Ventrikeldrainage.

\section{INFO}

\section{Chlorhexidin-Verbände}

Bislang gab es nur wenige Studien über den Einsatz von Chlorhexidingetränkten Verbänden für die Hauteintrittsstelle der EVD. Roethlisberger et al. wiesen in ihrer Arbeit nach, dass die Wiederbesiedelung der EVD-Hauteintrittsstelle mit Keimen durch den Einsatz Chlorhexidin-haltiger Verbände sicher reduziert werden konnte. Die Anzahl der kolonisierten Ventrikelkatheter war deutlich geringer, wenn auch nicht signifikant. Die Infektionsrate differierte nicht signifikant zwischen den beiden verglichenen Gruppen. Die Komplikation „Hydrozephalus" war in der Chlorhexidin-Gruppe aber niedriger [23]. Scheithauer et al. zeigten in ihrer Studie bei Patienten, deren EVD mit Chlorhexidin-haltigen Pflastern versorgt war, einen signifikanten Rückgang $(p=0,005)$ der Meningoventrikulitis-Rate [24].
Der Verband der Hauteintrittsstelle unterscheidet sich je nach Art des Zugangs: Getunnelte Liquordrainagen können sowohl mit Gazepflastern als auch mit ChlorhexidinPflastern problemlos versorgt werden ( $\bullet$ Abb.5). Dagegen gestaltet sich der Chlorhexidin-Verband bei einer Ventrikeldrainage, die über eine Schädelschraube eingeführt wird, extrem schwierig. In diesem Fall sollte ein Gazepflasterverband angelegt werden ( $\bullet$ Abb. $\mathbf{6})$.

\section{Kontrolle und Verbandwechsel}

Wie bei den ZVK gilt auch bei den Liquordrainagen: Das Gazepflaster muss täglich gewechselt werden, transparente Folienverbände müssen in der Regel nach 4, Chlorhexidin-haltige Pflaster nach 7 Tagen gewechselt werden. Es sind jeweils die Herstellerangaben zu beachten. Durchweichte und verunreinigte Verbände müssen sofort erneuert werden ( $\triangleright$ Abb. 7) [7].

Während der Liegedauer kann sich eine eingebrachte Schädelschraube (Gewinde) lockern. Dadurch ist die EVD dann nicht mehr sicher befestigt, was ein deutlich erhöhtes Infektionsrisiko darstellt. Daher muss täglich der feste Sitz der Schädelschraube kontrolliert werden.

Merke

Die tägliche Kontrolle der EVD-Eintrittsstelle und des Sitzes der Schädelschraube sind essenziell. Insbesondere ist auf Liquoraustritt zu achten.

\section{Wundverbände}

Nach den Atemwegsinfektionen sind die SSI die häufigsten nosokomialen Infektionen. In der Kolonchirurgie tritt eine SSI bei bis zu 30\% der operierten Patienten auf. In Europa gibt es keine Guidelines zur Vorbeugung von SSI.

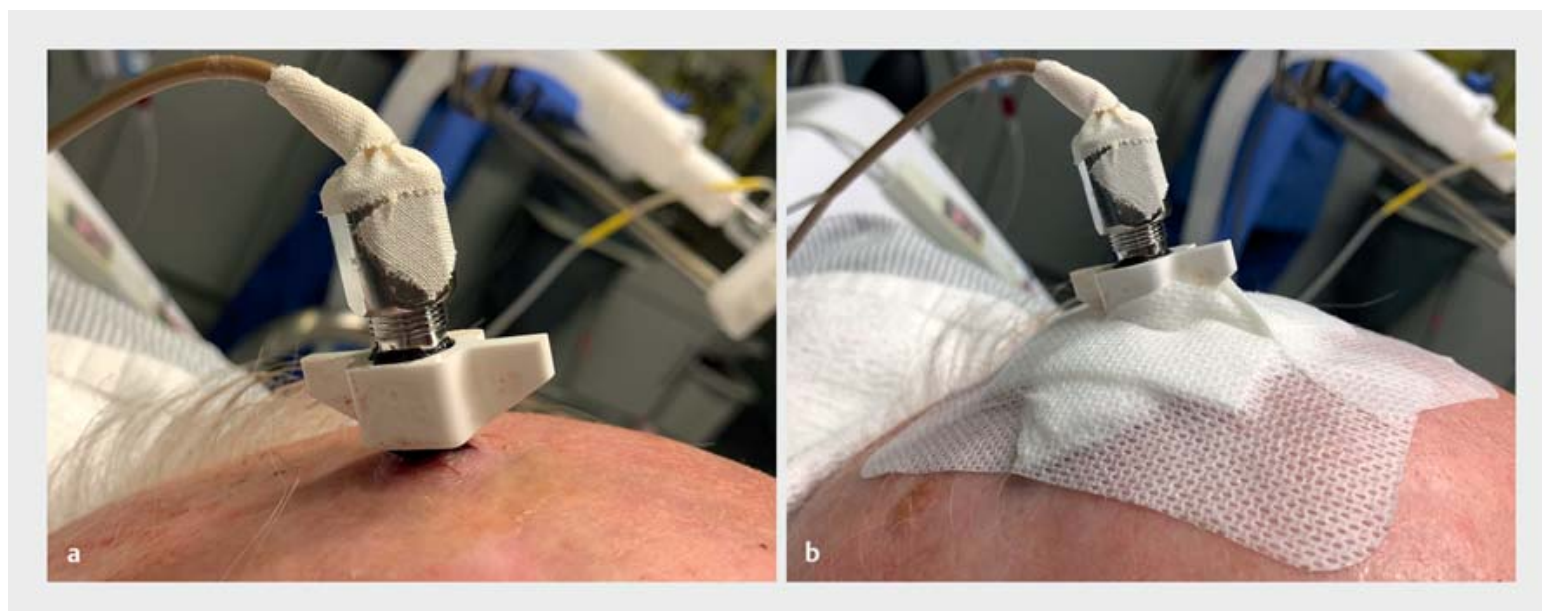

- Abb. 6 Über eine Schädelschraube eingeführte externe Ventrikeldrainage. a Schädelschraube ohne Verband. b Gazepflasterverband der Schädelschraube. 


\section{FALLBEISPIEL}

\section{Liquorinfektion}

Ein 46-jähriger Patient mit akutem Kopfschmerzereignis wird nach Intubation durch den Notarzt bei GCS 3 und Aspiration aufgenommen. Die Diagnostik ergibt: Subarachnoidalblutung aus Basilaris-Kopf-Aneurysma mit massivem Liquoraufstau durch Blut im III. und IV. Ventrikel. Nach EVD-Anlage (SpiegelbergSonde über Schädelschraube) erfolgt das Stent-gestützte Aneurysma-Coiling, danach Antikoagulation mit ASS und Clopidogrel.

Auf Intensivstation werden bei schwerer Oxygenierungsstörung (Horovitz-Index 100-120) eine kalkulierte Antibiose (Piperacillin/Tazobactam und Metronidazol) sowie die Lagerungstherapie (Bauchlage) unter ICP-Monitoring begonnen.

Tag 2: Liquor blutig, Liquor-Laktat mit 3,5 mmol// leicht erhöht und Liquor-Glukose 75 mg/dl (SerumGlukose 148 mg/dl). IL-6 im Liquor 3150 ng/l.

Tag 5: Der EVD-Verband ist durchgeschlagen; beim Verbandwechsel zeigt sich, dass die Schädelschraube gelockert ist und Liquor austritt. Eine Neuanlage ist unter doppelter Plättchenhemmung mit hohem Einblutungsrisiko verbunden. Nach gründlicher Desinfektion wird die Schädelschraube erneut fixiert. Tag 6: Der Patient entwickelt Fieber (Meningismus war schon vorher vorhanden); der Liquor ist weiterhin blutig und trübe. Anstieg des Liquor-Laktats auf $7,5 \mathrm{mmol} / \mathrm{l}$, Abfall der Liquor-Glukose auf $34 \mathrm{mg} / \mathrm{dl}$ (Serum 148 mg/dl), IL-6 im Liquor 87500 ng/l. Im Liquor wird Staphylococcus epidermidis nachgewiesen. Wegen nachgewiesener Liquorinfektion wird eine Antibiose mit Rifampicin und Vancomycin (systemisch und intrathekal) begonnen.

\section{Merke}

Ein stabiler Kreislauf, eine suffiziente Oxygenierung, Normothermie und ein strenges Transfusionsregime verringern die Entstehung von SSI [25, 26].

Bei chirurgischen Wunden sind die Wundränder in der Regel durch Nähte oder Klammern adaptiert und heilen meist primär ab. Die Wundverbände, die am Ende der Operation angelegt werden, entlasten die Wunde, schützen sie vor Kontamination und können austretendes Exsudat aufnehmen. Je nach Art und Lokalisation der Wunde bestehen die Wundverbände aus sterilen Gazepflastern, semipermeablen Pflastern oder aufgelegten sterilen Mullkompressen, die mit einer Klebefolie fixiert sind [25,27].

In einem Review von Dumville et al. [27] wurde die Häufigkeit des SSI-Auftretens in Abhängigkeit von verschiedenen Wundverbandarten sowie beim Verzicht auf einen Wundverband verglichen. Es konnte für keine Art des

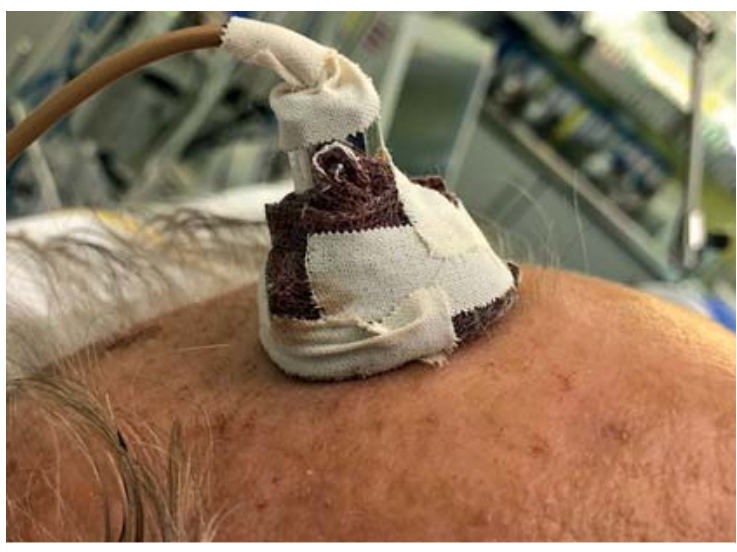

Abb. 7 Durchfeuchteter Gazepflasterverband.

Wundverbands eine signifikante Überlegenheit gezeigt werden. In einer tierexperimentellen Studie von Mana et al. [28] wurde bei anästhesierten Schweinen nach Setzen eines definierten Hautschnitts die dadurch erzeugte Wunde mit MRSA kontaminiert. Dann wurde die Wunde mit oder ohne ein Chlorhexidin-abgebendes Gelkissen verbunden. In der Chlorhexidin-Gruppe kam es zu einer signifikanten Reduktion der Wundinfektion.

\section{Kontrolle und Verbandwechsel}

Eine tägliche Kontrolle des Wundverbands ist obligat. Für den Zeitpunkt des ersten Wechsels eines Wundverbands gibt es keine Richtlinie. Mit Ausnahme von verschmutzten oder durchtränkten Wundverbänden, die immer zeitnah gewechselt werden müssen, sollte der erste Verbandwechsel nach ca. $48 \mathrm{~h}$ erfolgen. Eventuell vorhandene Fibrinbeläge und Nekrosen sollten aseptisch abgetragen werden. Die Wundspülung kann mit steriler isotoner Kochsalzlösung erfolgen, bei entzündeten Wunden ist die Spülung mit einem Antiseptikum (z. B. Octenidin) angezeigt [29].

\section{Merke}

Ein Wundverband muss jeden Tag kontrolliert werden. Jeder Verbandwechsel erfordert eine strenge Händehygiene und darf nie mit bloßen Händen erfolgen.

\section{Resistenzentwicklung}

\section{Chlorhexidin}

Die weitverbreitete Anwendung von Chlorhexidin im Gesundheitswesen birgt - wie in einer aktuellen Übersichtsarbeit von Kampf [32] dargestellt - erhebliche Risiken. In den letzten Jahren gibt es eine zunehmende Sorge über die Entwicklung von Resistenzen, nicht nur gegenüber Antibiotika, sondern auch gegen Antiseptika. Es konnte eine starke, stabile Resistenzentwicklung bei Bakterien- 


\section{INFO}

\section{Chlorhexidin-Nanopartikel}

Ein neuer Ansatz für Wundverbände sind auf Chlorhexidinmetaphosphat basierende Nanopartikel. Aus einer Reaktion von Chlorhexidindigluconat und Natriumhexametaphosphat entsteht ein stabiles Kolloid aus Chlorhexidin-basierten Nanopartikeln. Es bindet sich an Stoffe wie Metalloxide, Glas und elastische Wundverbände. Aus diesem Kolloid kann Chlorhexidin über 50 Tage hinweg freigesetzt werden [30].

In einer tierexperimentellen Studie wurde narkotisierten Mäusen eine Schnittwunde zugefügt und die Wunde mit Wundverbänden bedeckt. Die Verbände enthielten in der einen Gruppe Chorhexidindigluconat und in der anderen Gruppe Chlorhexidinmetaphosphat. Es zeigte sich in beiden Gruppen ein anhaltender antimikrobieller Effekt. Chlorhexidinmetaphosphat besaß ein ähnliches Wirkungsspektrum wie Chlorhexidindigluconat, zeigte jedoch eine niedrigere Toxizität. Die Wundheilung wurde nicht beeinflusst [31].

stämmen von Escherichia (E.) coli, Salmonella spp., Serratia marcescens und Pseudomonas aeruginosa gezeigt werden. Auch wurden Kreuzresistenzen z.B. mit Tetrazyklin, Gentamicin und Meropenem bei Bacteroides fragilis, Burkholderia cepacia und Salmonella spp. gefunden. Des Weiteren konnte eine verminderte Empfindlichkeit von E. coli gegenüber anderen Antiseptika - Triclosan und Benzalkoniumchlorid - gezeigt werden.

\section{Merke}

Unter Laborbedingungen konnte durch die Einwirkung von Chlorhexidin eine zunehmende Resistenzbildung von Keimen gegen Antiseptika nachgewiesen werden.

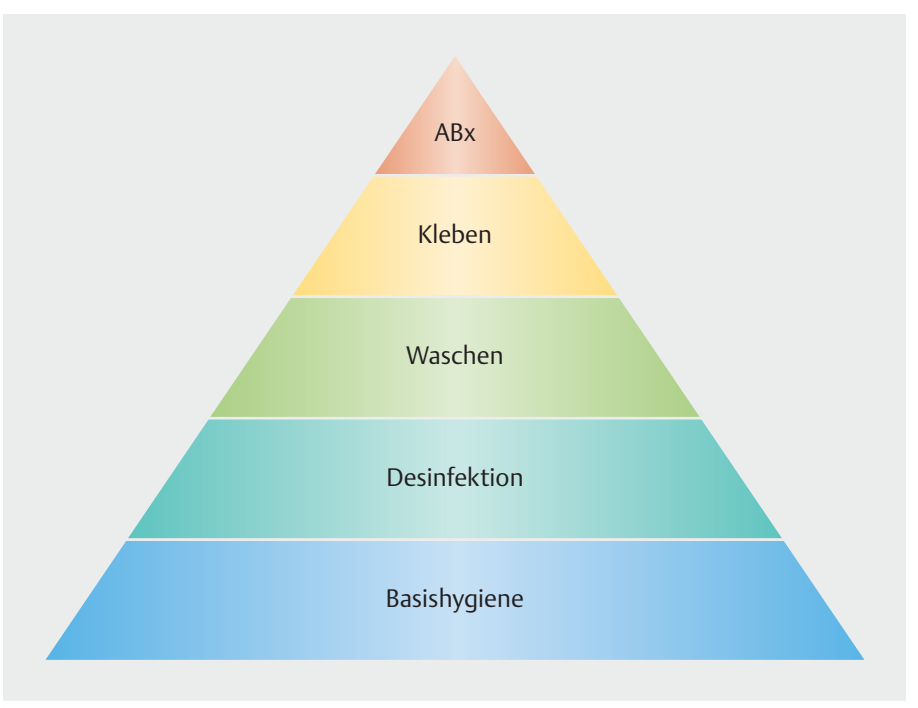

- Abb. 8 Infektionsprävention auf der Intensivstation. ABx: AntiinfektivaTherapie.
Besonders beunruhigend ist ein nachgewiesener horizontaler Gentransfer für eine Sulfonamid-Resistenz bei E. coli, was zu einer Resistenz innerhalb des gesamten Bakterienstamms führen kann. Zusätzlich konnten eine vermehrte Biofilmbildung bei Klebsiella pneumoniae und Serratia marcescens sowie eine erhöhte Affinität von Serratien an Polyethylen gefunden werden. Bei Vancomycinresistenten Enterokokken zeigte sich eine verminderte Empfindlichkeit gegenüber Daptomycin.

Wichtiger Bestandteil dieser Wirkungsabschwächung sind Effluxpumpen, die Stoffe aus der Bakterienzelle transportieren können [33]. Einige Effluxpumpen können nicht nur spezifisch einen Stoff transportieren, sondern eine große Menge verschiedener Stoffe aus dem Bakterium eliminieren. Somit kann es zu Kreuzresistenzen und Multiresistenzen kommen. Bei E. coli konnte nachgewiesen werden, dass sich unter Chlorhexidin-Einwirkung eine verminderte Empfindlichkeit des Bakteriums gegenüber Triclosan und Benzalkoniumchlorid entwickelte. Außerdem waren Salmonella spp. in der Lage, ihre Zellwandstruktur zu verändern und den Zellstoffwechsel vermehrt auf eine anaerobe Stoffwechsellage umzustellen. Eine Limitation der Studie von Kampf [32] ist, dass diese Ergebnisse unter Laborbedingungen zustande gekommen sind. Inwieweit diese Ergebnisse auf die Klinik übertragen werden können, bleibt bislang unklar. Jedoch wurden auch bei klinischen Isolaten (z. B. S. aureus) höhere MHK-Werte gefunden.

Es ist bekannt, dass Chlorhexidin mit einer geringen Menge Wasser vermengt eine niedrige bakterizide Wirkung besitzt. Inwieweit die Menge des Wassers, welches von der Haut freigesetzt wird, ein solches Mischungsverhältnis ergibt, ist nicht bekannt. Klar ist jedoch, dass eine subletale Chlorhexidin-Dosis für die Bakterien einen kontinuierlichen chemischen Stress darstellt und dass dieser Stress eine Resistenzbildung provozieren kann. Für die alkoholbasierten Händedesinfektionsmittel gibt es für den Zusatz von Chlorhexidin keine Evidenz. Wegen der Risiken der Resistenzentwicklung und Unverträglichkeitsreaktionen der Haut ist dieser Zusatz von der WHO auch nicht empfohlen [32].

\begin{abstract}
Merke
Chlorhexidin sollte nur dann eingesetzt werden, wenn für den Patienten ein deutlicher Vorteil zu erwarten ist. Eine engmaschige Surveillance muss gewährleistet sein.
\end{abstract}

\section{Octenidin}

Wegen der unspezifischen Wirkung auf die Bakterienzelle wurde lange Zeit davon ausgegangen, dass Octenidin keine relevante Resistenzbildung hervorruft. In einer neueren Arbeit konnten Dopcea et al. [34] aber zeigen: Auch unter Octenidin-Exposition kommt es zu einem Anstieg der MHK, zu einer verminderten Empfindlichkeit der Erre- 
ger gegenüber Antibiotika sowie zu einer verminderten Octenidin-Empfindlichkeit.

- Abb. 8 fasst den Aufbau und die Bedeutung der Maßnahmen der Infektionsprävention auf der Intensivstation zusammen.

\section{KERNAUSSAGEN}

- Basishygiene ist der Grundpfeiler, um Infektionen auf der Intensivstation zu verhindern. Sie verhindert das Übertragen von Keimen auf den Patienten. Wichtigste Einzelmaßnahme ist die sorgfältige Händehygiene.

- Desinfektion verhindert das Einbringen von Keimen in den Patienten bei der Insertion von Kathetern, bei Medikamentengabe über einliegende Katheter und Probenentnahmen aus den Devices. Die Anzahl der Abnahmen ist auf das Notwendige zu beschränken.

- Waschen mit Antiseptika reduziert die Anzahl der auf dem Patienten befindlichen Keime und kann somit die Gefahr der katheterassoziierten Infektionen reduzieren. Vor dem Hintergrund einer möglichen Resistenzbildung ist die Indikation streng zu stellen.

- Eine Dekontamination des Naso- und Oropharynx ist bei Hochrisikopatienten sinnvoll.

- Kleben reduziert die Keimzahl im Bereich von Katheter-Eintrittsstellen und verlangsamt die Geschwindigkeit einer erneuten Keimbesiedelung. Somit kann die Gefahr der katheterassoziierten Infektionen reduziert werden.

- Die prophylaktische Gabe von Antibiotika wegen einliegender Katheter ist nicht indiziert. Kommt es trotz Beachtung aller Punkte zu einem Keimnachweis, sollte nur bei Vorliegen einer Infektion, nicht aber bei einer Kolonisation eine Antibiose verabreicht werden.

- Die Indikation zur Anlage der Devices sollte streng gestellt werden; die Indikation, sie zu belassen, jeden Tag genau überprüft werden.

- Aufgrund gehäuft auftretender Meldungen über eine durch Chlorhexidin verursachte Resistenzentwicklung und unerwünschte Nebenwirkungen sollte das Antiseptikum nur in ausgewählten Situationen (nachgewiesener Vorteil für den Patienten) eingesetzt werden.

- Auch bei Octenidin sollte eine engmaschige Surveillance der Resistenzbildung erfolgen.

\section{Interessenkonflikt}

\section{Erklärung zu finanziellen Interessen}

Forschungsförderung erhalten: nein; Honorar/geldwerten Vorteil für Referententätigkeit erhalten: nein; Bezahlter Berater/interner Schulungsreferent/Gehaltsempfänger: nein; Patent/Geschäftsanteile/Aktien (Autor/Partner, Ehepartner, Kinder) an im Bereich der Medizin aktiven Firma: nein; Patent/ Geschäftsanteile/Aktien (Autor/Partner, Ehepartner, Kinder) an zu Sponsoren dieser Fortbildung bzw. durch die Fortbildung in ihren Geschäftsinteressen berührten Firma: nein.

Erklärung zu nichtfinanziellen Interessen

Die Autorinnen/Autoren geben an, dass kein Interessenkonflikt besteht.

\section{Autorinnen/Autoren}

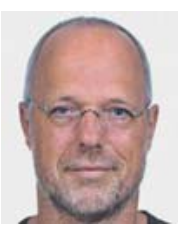

\section{Jochen Steiner}

Dr. med., Medizinstudium an der Eberhard Karls Universität Tübingen. Facharzt für Anästhesiologie, Zusatzbezeichnungen Intensivmedizin und Notfallmedizin. 1996-2018 Oberarzt der Neurochirurgischen Intensivstation an der Universitätsklinik Tübingen. Seit 11/2018 stellv. Leitender Oberarzt der anästhesiologisch-interdisziplinären Intensivstation an der Universitätsklinik Tübingen. Schwerpunkte: Neurochirurgische Intensivmedizin, Notfallmedizin.

\section{Korrespondenzadresse}

\section{Dr. med. Jochen Steiner}

Universitätsklinik Tübingen

Klinik für Anästhesie und Intensivmedizin

Hoppe-Seyler-Straße 3

72076 Tübingen

jochen.steiner@med.uni-tuebingen.de

\section{Wissenschaftlich verantwortlich} gemäß Zertifizierungsbestimmungen

Wissenschaftlich verantwortlich gemäß Zertifizierungsbestimmungen für diesen Beitrag ist Dr. med. Jochen Steiner, Tübingen.

\section{Literatur}

[1] Gastmeier P, Geffers C. Nosokomiale Infektionen in Deutschland: Wie viele gibt es wirklich? Dtsch med Wochenschr 2008; 133: $1111-1115$

[2] Nationales Referenzzentrum für Surveillance von nosokomialen Infektionen (NRZ). Deutsche nationale Punkt-Prävalenzerhebung zu nosokomialen Infektionen und Antibiotika-Anwendung 2016. Abschlussbericht. Im Internet: http://www. nrz-hygiene.de/fileadmin/nrz/download/pps2016/PPS_2016_ Abschlussbericht_20.07.2017.pdf; Stand: 14.04.2020

[3] Kommission für Krankenhaushygiene und Infektionsprävention (KRINKO) beim Robert Koch-Institut (RKI). Händehygiene in Einrichtungen des Gesundheitswesens. Empfehlung der Kommission für Krankenhaushygiene und Infektionsprävention (KRINKO) beim Robert Koch-Institut (RKI). Bundesgesundheitsbl 2016; 59: 1189-1220

[4] Kommission für Krankenhaushygiene und Infektionsprävention beim Robert Koch-Institut (RKI). Anforderungen an die Hygiene bei der Reinigung und Desinfektion von Flächen. 
Empfehlung der Kommission für Krankenhaushygiene und Infektionsprävention beim Robert Koch-Institut (RKI). Bundesgesundheitsbl 2004; 47: 51-61

[5] Institut für Hygiene und Umweltmedizin, Charité Universitätsmedizin Berlin. „Aktion Saubere Hände“, Compliance Beobachtungen - Referenzdaten 2017. Im Internet: http://www. aktion-sauberehaende.de/fileadmin/ash/downloads/pdf/ Compliance/201701_201712_ComplianceReference_final_ Alle_Stationen.pdf; Stand: 14.04.2020

[6] Kommission für Krankenhaushygiene und Infektionsprävention beim Robert Koch-Institut (RKI). Anforderungen bei Punktionen und Injektionen. Empfehlung der Kommission für Krankenhaushygiene und Infektionsprävention beim Robert KochInstitut (RKI). Bundesgesundheitsbl 2011; 54: 1135-1144

[7] Kommission für Krankenhaushygiene und Infektionsprävention (KRINKO) beim Robert Koch-Institut. Prävention von Infektionen, die von Gefäßkathetern ausgehen. Teil 1 - Nichtgetunnelte zentralvenöse Katheter Empfehlung der Kommission für Krankenhaushygiene und Infektionsprävention (KRINKO) beim Robert Koch-Institut. Bundesgesundheitsbl 2017; 60: 171-206

[8] Müller G, Langer J, Siebert J et al. Residual antimicrobial effect of chlorhexidine digluconate and octenidine dihydrochloride on reconstructed human epidermis. Skin Pharmacol Physiol 2014; 27: 1-8

[9] Assadian O. Octenidine dihydrochloride: chemical characteristics and antimicrobial properties. J Wound Care 2016; 25 (3 Suppl): S3-S6

[10] Kommission für Krankenhaushygiene und Infektionsprävention (KRINKO) beim Robert Koch-Institut. Prävention und Kontrolle Katheter-assoziierter Harnwegsinfektionen. Empfehlung der Kommission für Krankenhaushygiene und Infektionsprävention (KRINKO) beim Robert Koch-Institut. Bundesgesundheitsbl 2015; 58: 641-650

[11] McDonnell G, Russell AD. Antiseptics and disinfectants: Activity, action, and resistance. Clin Microbiol Rev 1999; 12: 147179

[12] Edmiston CE, Brudon B, Rucinski MC et al. Reducing the risk of surgical site infections: Does chlorhexidine gluconate provide a risk reduction benefit? Am J Infect Control 2013; 41(5 Suppl): S49-S55

[13] Gastmeier P, Kämpf KP, Behnke M et al. An observational study of the universal use of octenidine to decrease nosocomial bloodstream infections and MDR organisms. J Antimicrob Chemother 2016; 71: 2569-2576

[14] Frost SA, Alogso MC, Metcalfe L et al. Chlorhexidine bathing and health care-associated infections among adult intensive care patients: a systematic review and meta-analysis. Crit Care 2016; 20: 379

[15] Noto MJ, Domenico HJ, Byrne DW et al. Chlorhexidine bathing and healthcare-associated infections: a randomized clinical trial. JAMA 2015; 313: 369-378

[16] Lewis SR, Schofield-Robinson OJ, Rhodes S et al. Chlorhexidine bathing of the critically ill for the prevention of hospitalacquired infection. Cochrane Database Syst Rev 2019; (8): CD012248

[17] Li Bassi G, Senussi T, Aquilera Xiol E. Prevention of ventilatorassociated pneumonia. Curr Opin Infect Dis 2017; 30: 214220

[18] Segers P, Speekenbrink RGH, Ubbink DT et al. Prevention of nosocomial infection in cardiac surgery by decontamination of the nasopharynx and oropharynx with chlorhexidine gluconate. JAMA 2006; 296; 2460-2466

[19] D'Journo XB, Falcoz PE, Alifano M et al. Oropharyngeal and nasopharyngeal decontamination with chlorhexidine gluconate in lung cancer surgery: a randomized clinical trial. Intensive Care Med 2018; 44: 578-587
[20] Ullman A], Cooke ML, Mitchell $\mathrm{M}$ et al. Dressings and securement devices for central venous catheters (CVC). Cochrane Database Syst Rev 2015; (9): CD010367

[21] Yu K, Lu M, Meng Y et al. Chlorhexidine gluconate transparent dressing does not decrease central line-associated bloodstream infection in critically ill patients: A randomized controlled trial. Int J Nurs Pract 2019; 20: e12776

[22] Lozier AP, Sciacca RR, Romagnoli MF et al. Ventriculostomy-related infections: A critical review of the literature. Neurosurgery 2002; 5: 170-182

[23] Roethlisberger M, Moffa G, Fisch U et al. Effectiveness of a chlorhexidine dressing on silver-coated external ventricular drain-associated colonization and infection: a prospective single-blinded randomized controlled clinical trial. Clin Infect Dis 2018; 67: 1868-1877

[24] Scheithauer S, Schulze-Steinen H, Höllig A et al. Significant reduction of external ventricular drainage-associated meningoventriculitis by chlorhexidine-containing dressings: a beforeafter trial. Clin Infect Dis 2016; 62: 404-405

[25] Biffi R, Fattori L, Bertani E. Surgical site infections following colorectal cancer surgery: a randomized prospective trial comparing common and advanced antimicrobial dressing containing ionic silver. World J Surg Oncol 2012; 10: 94

[26] Kommission für Krankenhaushygiene und Infektionsprävention (KRINKO) beim Robert Koch-Institut. Prävention postoperativer Wundinfektionen. Empfehlung der Kommission für Krankenhaushygiene und Infektionsprävention (KRINKO) beim Robert Koch-Institut. Bundesgesundheitsbl 2018; 61: 448473

[27] Dumville JC, Gray TA, Walter C] et al. Dressings for the prevention of surgical site infection (Review). Cochrane Database Syst Rev 2014; (9): CD003091

[28] Mana TSC, Donskey C, Carty N et al. Preliminary analysis of the antimicrobial activity of a postoperative wound dressing containing chlorhexidine gluconate against methicillin-resistant Staphylococcus aureus in an in vivo porcine incisional wound model. Am J Infect Control 2019; 47: 1048-1052

[29] Kolasinski W. Surgical site infections - review of current knowledge, methods of prevention. Pol Przegl Chir 2018; 91 : 41-47

[30] Barbour ME, Maddocks SE, Wood NJ et al. Synthesis, characterization, and efficacy of antimicrobial chlorhexidine hexametaphosphate nanoparticles for applications in biomedical materials and consumer products. Int J Nanomedicine 2013; 8: 3507-3519

[31] Barbour ME, Maddocks SE, Grady H] et al. Chlorhexidine hexametaphosphate as a wound care material coating: antimicrobial efficacy, toxicity and effect on healing. Nanomedicine 2016; 11: 2049-2057

[32] Kampf G. Adaptive bacterial response to low level chlorhexidine exposure and its implications for hand hygiene. Microb Cell 2019; 6: 307-320

[33] Levy SB. Active efflux, a common mechanism for biocide and antibiotic resistance. J Appl Microbiol 2002; 92(Suppl): 65S715

[34] Nicolae Dopcea G, Dopcea I, Nanu A et al. Resistance and cross-resistance in Staphyloccocus sp. after prolonged exposure to different antiseptics. J Glob Antimicrob Resist 2019. doi:10.1016/j.jgar.2019.10.021

\section{Bibliografie}

DOI https://doi.org/10.1055/a-0967-1290

Anästhesiol Intensivmed Notfallmed Schmerzther 2020; 55: 368-380 @ Georg Thieme Verlag KG Stuttgart · New York ISSN 0939-2661 


\section{Punkte sammeln auf CME.thieme.de}

Diese Fortbildungseinheit ist in der Regel 12 Monate online für die Teilnahme verfügbar.

Den genauen Einsendeschluss finden Sie unter https://cme.thieme.de/.

Sollten Sie Fragen zur Online-Teilnahme haben, finden Sie unter https://cme.thieme.de/hilfe

eine ausführliche Anleitung. Wir wünschen viel Erfolg beim Beantworten

der Fragen!

Unter https://eref.thieme.de/CXDI47T oder über den QR-Code kommen Sie

direkt zur Startseite des Wissenstests.

VNR 2760512020158722930

\section{Frage 1}

Welche Aussage ist richtig? In einer Erhebung nosokomialer Infektionen im Jahre 2016 des Nationalen Referenzzentrums für Surveillance von nosokomialen Infektionen (NRZ) ...

A waren die häufigsten Infektionen Harnwegskatheter-assoziiert.

B waren die Harnwegsinfektionen in über $60 \%$ Harnwegskatheter-assoziiert.

C war die primäre Sepsis in unter $40 \%$ mit einem zentralen Venenkatheter (ZVK) assoziiert.

D war die Prävalenz nosokomialer Infektionen auf Normalstationen und Intensivstationen annähernd gleich.

E waren postoperative Wundinfektionen die häufigsten nosokomialen Infektionen.

\section{Frage 2}

Welcher der folgenden Ausrüstungsgegenstände wird zur Anlage eines zentralen Venenkatheters (ZVK) in Seldinger-Technik nicht benötigt?
A Mundschutz
B Kopfhaube
C steriler Kittel
D sterile Handschuhe
E OP-Schuhe

\section{Frage 3}

Welche Aussage zu Desinfektionsmitteln ist richtig?

A Zusätze von remanenten Antiseptika erhöhen die Wirkung eines alkoholischen Desinfektionsmittels.

B Ethanol und Propanol haben bei der Anwendung zur chirurgischen Händedesinfektion die gleiche Mindesteinwirkzeit.

C Ethanol wirkt stärker als Propanol.

D Octenidin ist ein Antiseptikum mit Remanenzwirkung.

E Chlorhexidin ist ein Alkohol.

\section{Frage 4}

Welche Aussage zur hygienischen Händedesinfektion ist richtig?

A Sie muss nur vor jedem Patientenkontakt durchgeführt werden.

B Sie muss nur nach jedem Patientenkontakt durchgeführt werden.

C Sie muss vor und nach jedem Patientenkontakt durchgeführt werden.

D Sie reduziert nur unwesentlich die Keimübertragung.

E Da es sich hierbei nicht um eine chirurgische Händedesinfektion handelt, muss die Mindesteinwirkzeit des Desinfektionsmittels nicht beachtet werden.

\section{Frage 5}

Welche Aussage ist richtig?

A Eine Antibiotikaprophylaxe ist der beste Schutz vor Deviceassoziierten nosokomialen Infektionen.

B Die Basishygiene ist einer der wichtigsten Bestandteile, um eine nosokomiale Infektion zu verhindern.

C Mit einem ZVK verbundene Dreiwegehähne müssen nur vor einer Medikamentengabe desinfiziert werden.

D Mit einem ZVK verbundene Dreiwegehähne müssen vor einer Blutentnahme nicht desinfiziert werden.

E Die Entnahmestellen für Liquorproben müssen nicht desinfiziert werden.

\section{Frage 6}

Welche Aussage zum Harnwegskatheter ist richtig?

A Die Anlage muss unter maximalen Barrieremaßnahmen erfolgen.

B Die Anlage muss aseptisch erfolgen.

C Die Reinigung des Genitales bei liegendem Harnwegskatheter muss mit einem alkoholischen Desinfektionsmittel erfolgen.

D Die Reinigung des Genitales bei liegendem Harnwegskatheter muss mit einem alkoholischen Desinfektionsmittel mit Remanenzzusatz erfolgen.

E Die Reinigung des Genitales sollte ausschließlich mit klarem Wasser erfolgen.

- Weitere Fragen auf der folgenden Seite... 


\section{Punkte sammeln auf CME.thieme.de}

Fortsetzung $\ldots$

\section{Frage 7}

Welche Aussage zu Harnwegskathetern ist richtig?

A Da es sich um eine Drainage handelt, muss die Entnahmestelle für eine Probe nicht desinfiziert werden.

B Die katheterassoziierte Harnwegsinfektion kommt im Vergleich zu den anderen Device-assoziierten Infektionen eher selten vor.

C Der Harnwegskatheter muss in regelmäßigem Abstand gewechselt werden, um eine Infektion zu vermeiden.

D Die Indikation muss täglich reevaluiert werden.

E Die Harnwegsinfektion ist ungefährlich, da sie auf die Harnwege beschränkt bleibt.

\section{Frage 8}

Welche Aussage zu Verbänden von ZVK-Eintrittsstellen ist falsch?

A Sie können mit sterilen Gazepflastern verbunden werden.

B Sie können mit sterilen semipermeablen Folienverbänden verbunden werden.

C Sie können mit sterilen Verbänden mit Chlorhexidin-haltigen Gelkissen verbunden werden.

D Verbände aus Gazepflaster müssen erst gewechselt werden, wenn sie durchfeuchtet sind.

E Semipermeable Folienverbände erleichtern die visuelle Kontrolle der Katheter-Eintrittsstelle.

\section{Frage 9}

Welche Aussage zu Verbänden mit Chlorhexidin-haltigem Gelkissen ist richtig?

A Sie verhindern zuverlässig eine katheterassoziierte Infektion.

B Sie sind als Verband der Hauteintrittsstelle einer externen Ventrikeldrainage wegen der Nähe zum Gehirnparenchym nicht zulässig.

C Ihre Verwendung ist immer unbedenklich.

D Sie reduzieren die Geschwindigkeit der Keimwiederbesiedelung an der ZVK-Eintrittsstelle.

E Sie müssen erst beim Wechsel (nach ca. 7 Tagen) kontrolliert werden.

\section{Frage 10}

Welche Aussage ist richtig?

A Da Chlorhexidin eine unspezifische antibakterielle Wirkung besitzt, kann es bedenkenlos eingesetzt werden.

B Da Chlorhexidin eine unspezifische antibakterielle Wirkung besitzt, sind bislang keine Resistenzentwicklungen bekannt.

C Unverträglichkeitsreaktionen auf Chlorhexidin sind bislang nicht aufgetreten.

D Bislang ist keine Resistenzbildung von mit Octenidin behandelten Keimen bekannt.

E Chlorhexidin kann zur Ganzkörperwaschung bei MRSA-Keimträgerschaft eingesetzt werden. 